\title{
Psychophysical scales of age based on marriage frequencies
}

\author{
ERIC W. HOLMAN \\ University of California, Los Angeles, California 90024
}

\begin{abstract}
The frequency of marriage between women of one age and men of another was assumed to depend upon the overall tendencies of women and men of the corresponding psychological ages to marry, multiplied by a nonincreasing function of the absolute difference between the psychological ages. Using this model and standard nonmetric scaling techniques, scales of psychological age as a function of physical age were inferred from published marriage frequencies for 1951 and 1959. Psychological age was well approximated by the cube root of the difference between physical age and a threshold age of about 12 years for women and 14 years for men.
\end{abstract}

Probably the oldest model for psychophysical scaling is based on the assumption that perceptually equal differences between stimuli correspond to numerically equal differences between their scale values. Fechner (1860) introduced this assumption for just noticeable differences and concluded that psychophysical functions were logarithmic. Thurstone (1927) later extended Fechner's model to social stimuli by assuming that disagreements between subjects in comparative judgments were analogous to confusions within a subject, but he did not derive psychophysical functions because his stimuli were not measured physically. The more recent development of nonmetric scaling techniques by Kruskal (1964a, 1964b) and Shepard (1962a, 1962b) has allowed the same model to be used for stimuli that were not confusable in any sense. Applications of these techniques to judgments of difference or similarity between pairs of stimuli have typically yielded psychophysical scales that were power functions with exponents less than unity (Rule, Curtis, \& Markley, 1970; Schneider, Parker, Valenti, Farrell, \& Kanow, 1978).

The present paper used the same psychophysical model and nonmetric techniques to scale a continuum not previously considered from a psychophysical point of view, age of people. Perceptual differences between ages were inferred not from experimental data on confusions or judgments, but from demographic data on frequencies of marriage between people of various ages. The present application is thus similar to Thurstone's in using social stimuli and data from large numbers of subjects, but different in using stimuli with a well-defined physical attribute that allows the construction of psychophysical functions.

Computer time for this work was provided by the UCLA Office of Academic Computing. I thank Thomas D. Wickens for his helpful suggestions.

\section{DATA AND ANALYSIS}

The yearly volumes of Vital Statistics of the United States have included, since 1949 , tables of marriages by age of each spouse in states where such information was collected. The tables included the actual numbers of marriages through 1959 , but only estimates based on samples starting in 1960 . The present paper analyzed the data from 1951 and 1959 (United States Department of Health, Education, and Welfare, 1951,1959 ), in order to compare two years during the period when the most complete data were reported. The 1951 data included 437,381 marriages in 21 states, and the 1959 data included 774,003 marriages in 29 states.

The published tables for both years partitioned ages into 1-year intervals from ages 15 through 34 years, and into five-year intervals from ages 35 through 74 years. To compensate in the present analysis for the unequal lengths of the intervals, the numbers of marriages in 1-year intervals cross-classified with 5year intervals were divided by 5 , and the numbers of marriages in 5-year intervals cross-classified with 5year intervals were divided by 25 . The resulting numbers are henceforth denoted $n(x, y)$, where $x$ is the midpoint of the woman's age interval and $y$ is the midpoint of the man's age interval.

Unfortunately, the number of marriages between women of one age and men of another depends not only upon the psychological difference between their ages, but also upon the number of single women and men of those ages, their interest in marriage, and their attractiveness as marriage partners. Various models for these overall effects of age have been proposed by demographers to describe the relation between the age distribution of a population and the age distribution of marriages within that population. Keyfitz (1972) compared several such models and concluded that a simple multiplicative model is about 
as consistent with the data as any other. This model assumes functions $w(x), m(y)$, and $s(x, y)$, such that

$$
n(x, y)=w(x) \cdot m(y) \cdot s(x, y) .
$$

The functions $w(x)$ and $m(y)$ represent, respectively, the overall tendencies of women of age $x$ and men of age $y$ to marry, and the function $s(x, y)$ represents the compatibility between women of age $x$ and men of age $y$. The present paper sought two scales of psychological age, $\psi_{\mathrm{w}}$ for women and $\psi_{\mathrm{m}}$ for men, such that

$$
\begin{gathered}
\mathrm{w}(\mathrm{x})=\mathrm{f}\left[\psi_{\mathrm{w}}(\mathrm{x})\right], \\
\mathrm{m}(\mathrm{y})=\mathrm{f}\left[\psi_{\mathrm{m}}(\mathrm{y})\right], \\
\mathrm{s}(\mathrm{x}, \mathrm{y})=\mathrm{g}\left[\left|\psi_{\mathrm{w}}(\mathrm{x})-\psi_{\mathrm{m}}(\mathrm{y})\right|\right],
\end{gathered}
$$

where $f$ is a nonnegative function and $g$ is a nonnegative nonincreasing function. The use of the same function $f$ for both sexes in Equations 2 and 3 reflects an assumption that the overall tendencies of women and men to marry are the same if their psychological ages are the same. The properties of the function $\mathrm{g}$ in Equation 4 reflect the psychophysical assumption that the compatibility between women and men tends to decrease as their psychological ages become more different.

These functions were disentangled from the data by estimating first the $w(x)$ and $m(y)$, then the $s(x, y)$, and finally the $\psi_{\mathrm{w}}(\mathrm{x})$ and $\psi_{\mathrm{m}}(\mathrm{y})$. First, Equations 1 through 4 imply that if $\psi_{\mathrm{w}}(\mathrm{x})=\psi_{\mathrm{m}}(\mathrm{y})$, then

$$
w(x)=m(y)=\sqrt{n(x, y) / g(0)} .
$$

Moreover, since $g$ is nonincreasing, $n(x, y)$ would be expected to attain a relative maximum whenever $\psi_{\mathrm{w}}(\mathrm{x})=\psi_{\mathrm{m}}(\mathrm{y})$. Since the locus of maxima in the matrix of the $n(x, y)$ was approximately parallel to the positive diagonal, the maxima were estimated from the data by searching along lines parallel to the negative diagonal, that is, by finding for each $\mathrm{z}$ the maximum value of $n(x, y)$, such that $x+y=z$. With $g(0)$ arbitrarily set at 100 , Equation 5 then yielded an estimate of $w(x)$ and $m(y)$ for each maximum or, with occasional interpolation, two estimates of $w(x)$ and $m(y)$ for each $x$ and $y$, whose means were then used as the final estimates of $w(x)$ and $m(y)$. These estimates were then substituted into Equation 1 to produce an estimate of $s(x, y)$ from each $n(x, y)$.

The psychophysical functions $\psi_{\mathrm{w}}$ and $\psi_{\mathrm{m}}$ could then be inferred from the estimated $s(x, y)$ by means of Equation 4. Although Equation 4 refers to compatibility between women and men rather than confusion between stimuli, it is mathematically very similar to Fechner's original psychophysical model. The only difference is that Equation 4 includes separate psychophysical functions for women and men rather than a single function for all stimuli. This generalization to two psychophysical functions has been explored theoretically by Coombs (1950) in his unfolding model and is available as an option in KYST, an extension of the multidimensional scaling programs of Kruskal (1964a, 1964b) and Young and Torgerson (1967). The program estimates the values of both functions on the same interval scale; that is, the scale values are (approximately) unique except for a single arbitrary origin and a single arbitrary unit.

The scale values were therefore found by applying KYST to the $s(x, y)$. Stress Formula 2 was used to avoid the degenerate solutions allowed by Formula 1 with the unfolding model. The $s(x, y)$ were weighted proportionally to $s(x, y)+1$, because otherwise the scale values would have been determined mainly by the longer distances, which, in this case, depended upon very few marriages or none at all. Several starting configurations were used to avoid the local minima common in unidimensional scaling.

\section{RESULTS AND DISCUSSION}

The best scaling solutions had stress values of .247 and .219 for the 1951 and 1959 data, respectively. These stress values are substantially higher than those previously reported for judgment data (Rule et al., 1970; Schneider et al., 1978). Although the high stress values may reflect an inferior fit of the model to the data, they can also be attributed at least in part to three methodological features of the present analysis not shared by the earlier studies. First, Stress Formula 2 gives higher stress values than does the more commonly used Formula 1 . Second, the weighting function used here gave little weight to the low data values that could be trivially fit with low stress. Third, the number of data values was much larger in the present study than in the earlier ones, both absolutely and in comparison with the number of scale values.

Figure 1 presents the obtained scale values as a function of physical age for both sexes in both years. The psychophysical functions are quite similar for the two sexes, except that the scale values for women are consistently higher than those for men; this difference reflects the fact that the wife is younger than the husband in most marriages. The functions are also very similar in the two years, except that the difference between the sexes is slightly greater in 1951 than in 1959. All the functions are clearly negatively accelerated at low physical ages and more nearly linear at high physical ages.

In order to compare the observed psychophysical functions with the logarithmic function suggested by Fechner and the power function suggested by later authors, Figure 2 presents the same scale values as a function of physical age on a logarithmic scale. A 


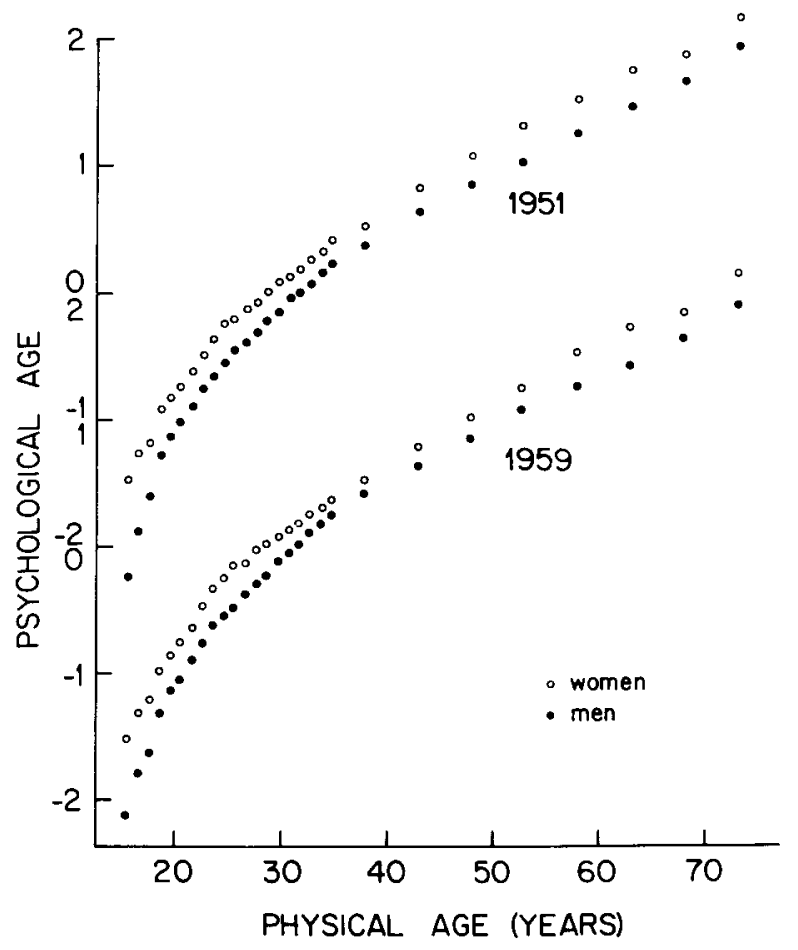

Figure 1. Psychological age as a function of physical age, for women and men, in 1951 (upper figures on ordinate) and 1959 (lower figures on ordinate).

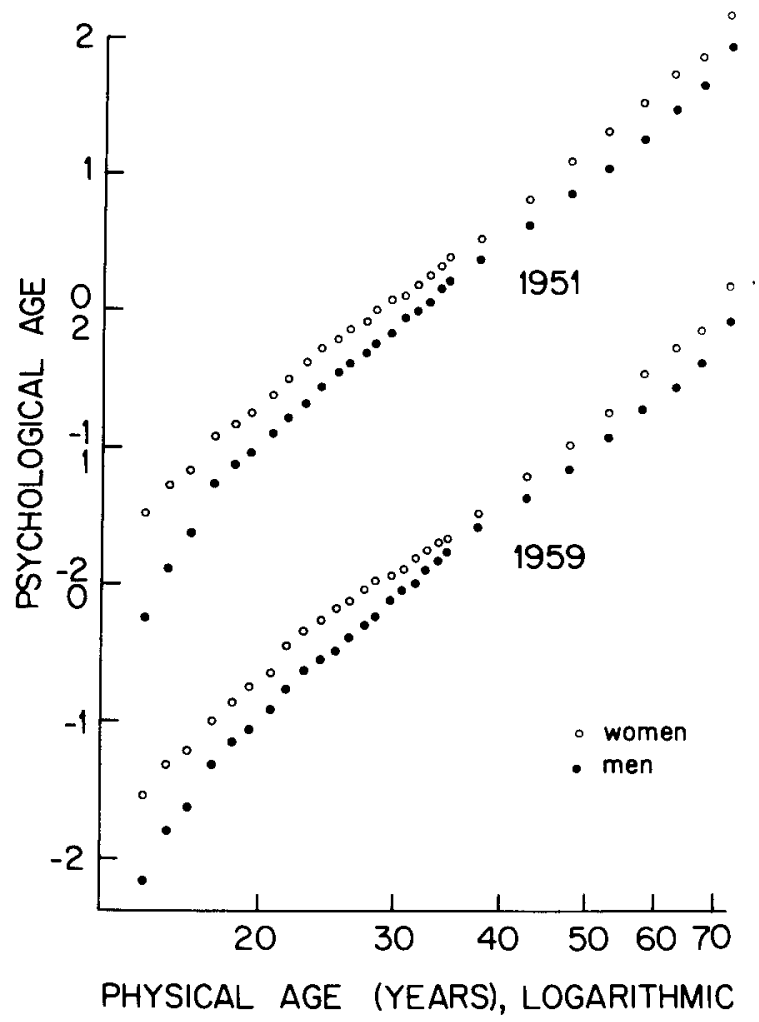

Figure 2. Psychological age as a function of physical age on a logarithmic scale, for women and men, in 1951 (upper figures on ordinate) and 1959 (lower figures on ordinate). logarithmic psychophysical function would be linear in this figure, and a power function would be positively accelerated. The actual functions are still negatively accelerated at low physical ages, and now slightly positively accelerated at high physical ages. Thus, both logarithmic and power psychophysical functions can be rejected for the present data.

To straighten a psychophysical function that is negatively accelerated at low values of the physical stimulus, Stevens (1959) has suggested that a constant, interpreted as a threshold, be subtracted from the value of the physical stimulus before the psychophysical function is applied. For a logarithmic function, however, a constant subtracted from physical age would increase the positive acceleration already present at high ages in Figure 2, as well as decreasing the negative acceleration at low ages. Thus, a logarithmic psychophysical function with a threshold can be rejected for the present data.

The remaining possibility, which is not obviously inconsistent with Figures 1 and 2, is a power function with a threshold. Such a function can be represented as follows, for women and men, respectively:

$$
\begin{aligned}
& \psi_{w}(x)=k\left(x-t_{w}\right)^{\gamma}+a, \\
& \psi_{m}(y)=k\left(y-t_{m}\right)^{a}+a .
\end{aligned}
$$

The constants a and $\mathrm{k}$ specify the arbitrary origin and unit of the scale values; $t_{w}$ and $t_{m}$ are the age thresholds for women and men; and $\gamma$ and $\alpha$ are the exponents for women and men. These equations imply the following relationships:

$$
\begin{aligned}
& \log \left[\psi_{w}(x)-a\right]=\gamma \cdot \log \left(x-t_{w}\right)+\log k, \\
& \log \left[\psi_{m}(y)-a\right]=\alpha \cdot \log \left(y-t_{m}\right)+\log k .
\end{aligned}
$$

The parameters in Equations 6 were estimated in two stages. First, the parameter estimation program of Wickens (1967) was used to find values of $a, t_{w}$, and $t_{m}$ that maximized the mean of the squared productmoment correlations $\left(r^{2}\right)$ between $\log \left[\psi_{w}(x)-a\right]$ and $\log \left(x-t_{w}\right)$ and between $\log \left[\psi_{m}(y)-a\right]$ and $\log$ $\left(y-t_{m}\right)$; these correlations do not involve the remaining parameters. Next, the estimated values of $a, t_{w}$, and $t_{m}$ were substituted back into Equations 6 , and the exponents $\gamma$ and $\alpha$ were estimated as the slopes of the functions; it was not necessary to estimate the arbitrary unit $k$. Table 1 presents the estimates of the thresholds and exponents and the mean squared correlations for both years. Figure 3 presents $\psi_{w}(x)-a$ and $\psi_{\mathrm{m}}(\mathrm{y})-\mathrm{a}$ on a logarithmic scale as functions of $x-t_{w}$ and $y-t_{m}$ on a logarithmic scale for both years.

The psychophysical functions are quite close to linear in Figure 3, and the proportion of variance explained by linear functions is very high. Therefore, a power function with a threshold gives an adequate fit to the present data. The fact that the estimated ex- 
Table 1

Parameter Values and Mean Squared Correlations for Power Functions With Thresholds

\begin{tabular}{cccccccc}
\hline & \multicolumn{2}{c}{ Threshold } & & \multicolumn{2}{c}{ Exponent } & \\
\cline { 2 - 3 } & Women & Men & & Women & Men & $\mathrm{r}^{2}$ \\
\hline 1951 & 12.06 & 14.80 & & .355 & .332 & .9985 \\
1959 & 12.49 & 14.43 & & .336 & .333 & .9983 \\
\hline
\end{tabular}

ponents are substantially greater than zero provides additional evidence against a logarithmic function with a threshold. Indeed, the present exponents are well within the range of exponents previously obtained by scaling judgments of similarity or difference between stimuli on continua other than age (Rule et al., 1970; Schneider et al., 1978).

The threshold ages turn out to be plausible estimates of the minimum ages at which marriages ever occur in the United States. Moreover, the lower thresholds for women than for men account for the higher scale values for women than for men of the same physical age, and the greater difference between

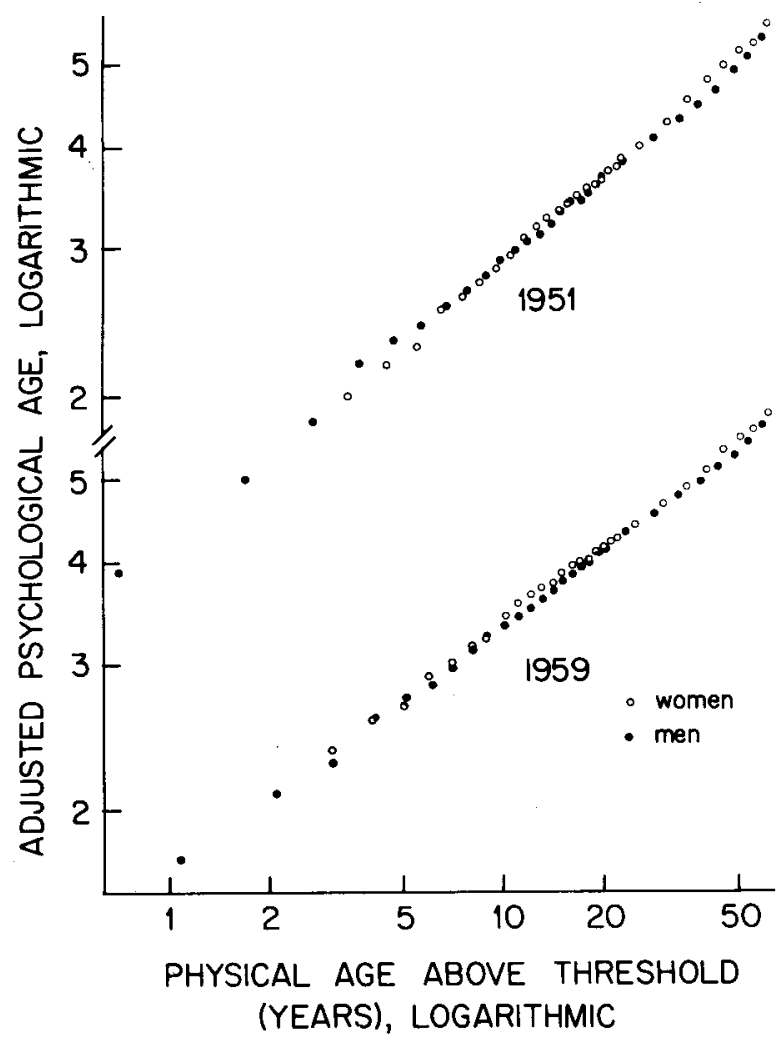

Figure 3. Adjusted psychological age on a logarithmic scale as a function of physical age above threshold on a logarithmic scale, for women and men, in 1951 (figures above break on ordinate) and 1959 (figures below break on ordinate). Note that, to obtain actual physical age, the appropriate threshold from Table 1 must be added to the figures on the abscissa. the thresholds in 1951 than in 1959 accounts for the greater differences in scale values between the sexes in the earlier year. Once the different thresholds are subtracted from physical age, all the exponents are nearly the same, and the four psychophysical functions reduce, for all practical purposes, to a single one.

In conclusion, the present analysis extended Thurstone's application to social stimuli of Fechner's model by deriving a psychophysical function for one set of such stimuli. The function itself, however, was not Fechner's logarithmic function, but rather was a power function, approximately the cube root. Thus, Fechner's model can be applied to marriages as well as to similarity judgments and confusions, but the model leads to Fechner's law only for confusions.

\section{REFERENCES}

Coomes, C. H. Psychological scaling without a unit of measurement. Psychological Review, 1950, 57, 145-158.

Fechnen, G. T. Elemente der Psychophysik. Leipzig: Breitkopf und Hartel, 1860. (Reissued, Amsterdam: E. J. Bonset, 1964.)

KeYfitz, $N$. The mathematics of sex and marriage. In L. M. LeCam, J. Neyman, \& E. L. Scott (Eds.), Proceedings of the Sixth Berkeley Symposium on Mathematical Statistics and Probability (Vol. 4). Berkeley: University of California Press, 1972.

KRUSKaL, J. E. Multidimensional scaling by optimizing goodness of fit to a nonmetric hypothesis. Psychometrika, 1964, 29, 1-28. (a)

Kruskal, J. E. Nonmetric multidimensional scaling: A numerical method. Psychometrika, 1964, 29, 115-130. (b)

Rule, S. J., Curtis, D. W., \& Markley, R. P. Input and output transformations from magnitude estimation. Journal of Experimental Psychology, 1970, 86, 343-349.

Schneider, B., Parker, S., Valenti, M., Farrell, G., \& KAnow, G. Response bias in category and magnitude estimation of difference and similarity for loudness and pitch. Journal of Experimental Psychology: Human Perception and Performance, 1978, 4, 483-496.

ShePARd, R. N. The analysis of proximities: Multidimensional scaling with an unknown distance function. I. Psychometrika, $1962,27,125-140$. (a)

ShePARD, R. N. The analysis of proximities: Multidimensional scaling with an unknown distance function. II. Psychometrika, $1962,27,219-246$. (b)

Stevens, S. S. Tactile vibration: Dynamics of sensory intensity. Journal of Experimental Psychology, 1959, 57, 210-218.

Thurstone, L. L. A law of comparative judgment. Psychological Review, 1927, 34, 273-286.

United States Department of Health, Education, and WeLfare. Vital statistics of the United States, 1951, Vol. 1, Table 2, p. 78.

United States Department of Health, Education, and Welfare. Vital statistics of the United States, 1959, Vol. 1, Table 8, p. 67.

Wickens, T. D. Parameter estimation in Markov chain learning models. Unpublished master's thesis, Brown University, 1967.

Young, F. W., \& Tongerson, W. S. TORSCA: A Fortran IV program for Shepard-Kruskal multidimensional scaling analysis. Behavioral Science, 1967, 12, 498.

(Received for publication September 5, 1980; revision accepted April 3, 1981.) 